\title{
BMJ Open Prescribing for Australians living with dementia: study protocol using the Delphi technique
}

\author{
Amy Page, ${ }^{1}$ Kathleen Potter, ${ }^{1}$ Rhonda Clifford, ${ }^{1}$ Andrew McLachlan, ${ }^{2}$ \\ Christopher Etherton-Beer ${ }^{1}$
}

To cite: Page A, Potter $\mathrm{K}$, Clifford R, et al. Prescribing for Australians living with dementia: study protocol using the Delphi technique. BMJ Open 2015;5:e008048. doi:10.1136/bmjopen-2015008048

- Prepublication history and additional material is available. To view please visit the journal (http://dx.doi.org/ 10.1136/bmjopen-2015008048).

Received 25 February 2015 Revised 10 July 2015 Accepted 16 July 2015

\section{CrossMark}

\footnotetext{
${ }^{1}$ School of Medicine and Pharmacology, University of Western Australia, Perth, Australia

${ }^{2}$ Faculty of Pharmacy, University of Sydney, Sydney, New South Wales, Australia
}

Correspondence to Amy Page;

Amy.page@uwa.edu.au

\section{ABSTRACT}

Introduction: Prescribing is complicated for people living with dementia, and careful consideration should be given to continuing and initiating all medicines. This study aims to elicit opinion and gain consensus on appropriate medicine use for people living with dementia in Australia to create a consensus-based list of explicit prescribing criteria.

Methods and analysis: A Delphi technique will be used to develop explicit criteria of medication use in adults aged 65 years and above. An interdisciplinary panel of Australian experts in geriatric therapeutics will be convened that will consist of a minimum of 10 participants. To develop the consensus-based criteria, this study will use an iterative, anonymous, multistaged approach with controlled feedback. Round 1 questionnaire will be administered, and subsequently qualitatively analysed. The round 1 results will be fed back to the panel members, and a round 2 questionnaire developed using questions on a five-point Likert scale. This process will repeat until consensus is developed, or diminishing returns are noted.

Ethics and dissemination: All participants will be provided with a participant information sheet, and sign a written consent form. Ethical approval has been granted from the University of Western Australia's Human Research Ethics Committee (HREC) (reference: RA/4/1/7172). We expect that data from this study will result in a paper published in a peerreviewed clinical journal and will also present the results at conferences.

\section{INTRODUCTION}

Dementia is a life-limiting disease with an average survival time of less than 5 years from diagnosis. ${ }^{1}{ }^{2}$ It is the third leading cause of death and the leading cause of disability burden in adults aged 65 years and above in Australia. ${ }^{34}$ Comorbidities and polypharmacy are common in people living with dementia, though data are scarce for medicine safety, tolerability and efficacy in this population.

Dementia is an umbrella term describing a collection of diseases characterised by

\section{Strengths and limitations of this study}

- Explicit prescribing criteria for medication use in older adults living with dementia will be developed by an interdisciplinary expert panel using a validated consensus method.

- The Delphi technique is a technique to make use of the available information by summarising existing knowledge and experience of prescribing from an interprofessional panel of experts in geriatric practice.

- As the Delphi technique is a consensus development method, it is not a method to create new evidence. Further, work will be needed to assess the usefulness of the criteria.

progressive neurological decline affecting language, memory, perception, personality and cognitive skills. ${ }^{3}$ The term dementia refers to over 100 illnesses and conditions, of which the most common are Alzheimer's, vascular, Lewy body and frontotemporal dementia. ${ }^{3}$ The aetiology, progression and manifestation of dementia may vary depending on the dementia type.

People living with dementia have as many or more comorbidities as their peers (defined as cognitively intact people of a comparable age) though how these comorbidities affect function and survival is uncertain. ${ }^{5-9}$ As they are taking a mean of 5 or more medicines daily, it appears these comorbidities are generally treated pharmacologically. ${ }^{9-11}$ People living with dementia are also more likely than their peers to use certain medicine classes, such as antihypertensives, laxatives, diuretics, antidepressants and antipsychotics. ${ }^{12} 13$

It is largely unknown if medicines for comorbidities have a similar safety profile and efficacy in people living with dementia compared to their peers or younger people. Age-related pharmacokinetic changes occur in all older people. ${ }^{14-16}$ Further, people with dementia are excluded from $85 \%$ of 
published clinical trials due to their inability to give informed consent. ${ }^{17}$ An altered blood-brain permeability in dementia means they may be more sensitive to neurological and cognitive effects of medicines than their peers. ${ }^{18}$ These pharmacokinetic changes are additional to the drug-disease interactions that occur in dementia. ${ }^{19}$ Further, the tendency for people with dementia to under-report disease-related symptoms means that it is likely they also under-report side effects. $^{20}$

The appropriateness of medicine use in this life-limiting condition is poorly understood despite the frequency of comorbidities and medicine use. Studies of antihypertensives, hypoglycaemics, statins and antiinflammatories mainly assess their ability to delay dementia onset. ${ }^{21-26}$ After dementia onset, medicine appropriateness to manage comorbidities is poorly understood. Further, preventive treatments may require a longer time to benefit than is available in a life-limiting condition, ${ }^{27}$ or may target treatment goals that are not relevant to the individual or their families. $^{28}$

Existing research for medicine use in people living with dementia focuses on treatments that prevent or delay dementia onset and/or progression, or to manage the dementia-specific symptoms such as the transient neuropsychiatric or behavioural symptoms common in people living with dementia. ${ }^{30} 31$ Evidence for the efficacy of these medicines is conflicting, ${ }^{32} 33$ and the risks of some, such as antipsychotics and benzodiazepines, make them potentially inappropriate in this population. ${ }^{34}$

Prescribing is complicated for people living with dementia, and careful consideration should be given to continuing and initiating all medicines. Prescribing decisions for people living with dementia are often based on data collected in younger adults or peers, which may not be generalisable or relevant to this population. The existing explicit prescribing criteria developed for older people do not account for the additional complexities of dementia or its life-limiting nature. ${ }^{35-39}$ An explicit prescribing criteria specifically for people living with dementia is needed.

The objective of this study is to elicit opinion and gain consensus on appropriate medicine use for people living with dementia in Australia. The intended outcome is to create a consensus-based list of explicit prescribing criteria for people living with dementia in Australia. This list of explicit prescribing criteria for people living with dementia that will state a list of preferred, or indicated, medicines or classes, as well as medicines or medicine classes that are potentially inappropriate. Identified medicines or classes may include one or more qualifying statements: medicines that are conditionally inappropriate (ie, in drug-drug combinations or disease states), options for minimising the risks associated with medicine use, preferred therapeutic alternatives, dose range (minimum dose or maximum dose) appropriate for this population.
METHOD

\section{Justification for study design}

This study will use the Delphi technique. For most medicines, there are insufficient strong high-quality data to develop comprehensive evidence-based explicit criteria prescribing in people living with dementia. We have chosen to use a Delphi technique as the most appropriate study design to develop explicit criteria that are consensus based where an insufficient quantity or grade of evidence exists to develop evidence-based criteria. ${ }^{40}$ Other methods commonly used to achieve consensus (eg, a focus group) would not be feasible as the expert panel will represent diverse geographical locations within Australia that would be impractical and costly to meet in person. ${ }^{41}$ Strengths of the Delphi technique that made it suitable for our study include participants anonymity (to each other, though not the researcher), and no individual could dominate discussion.

\section{Research steering group}

A research steering group will be formed to undertake this research. They will be responsible for preparing and circulating the content of the Delphi rounds. The research steering group will comprise of the investigators, with backgrounds including pharmacy, general practice, geriatrics, and pharmacology. The research steering group will not participate in the surveys; rather, they will supervise and monitor the process.

\section{Selection and identification of the expert panel}

Heterogeneity of the expert panel is important. The panel will consist of clinical-based or research-based experts with relevant backgrounds, for example, pharmacists, pharmacologists, geriatricians, general practitioners, palliative care physicians and other identified, relevant experts. Other medical experts including gastroenterologists, cardiologists, urologists, psychiatrists and endocrinologists may be included if they have relevant expertise in people living with dementia. Allied health professionals may be included if they have relevant expertise in treating people living with dementia, and are familiar with the medicines used.

Participants will be included if they are willing to participate and declare any conflicts of interest. Potential participants must meet one or more of the following criteria: authored one or more peer-reviewed papers relevant to pharmacotherapy used by people living with dementia (first, second or last author published within the past 10 years); or practiced in a field relevant to pharmacotherapeutics used by people living with dementia for five or more years post-graduation; or hold postgraduate qualifications or current credentials relevant to geriatric pharmacotherapeutics (eg, a geriatrician, palliative care physician, or a pharmacist credentialled with the Certifi cate of Geriatric Pharmacy), or recognised as an expert in the field by peers (eg, invitation to participate in a relevant symposium, focus group, conference or other event, or a 
member of relevant organisation, or received a personalised invitation letter to participate in the study). Participants will be excluded if they have insufficient experience in a relevant field or are unable to commit to be available for the entire process.

\section{Recruitment}

Participants will be identified using a multipronged approach (see figure 1) including self-nomination, identification of individuals by the authors. Individuals identified will be sent a personalised letter of invitation.

\section{Experts identified from the literature (route A)}

We will identify Australian researchers with expertise in geriatric pharmacotherapeutics by searching and crossreferencing recent publications in the field. Publications need to be relevant to medicine use in people living with dementia, and published in the past 10 years. These authors will be contacted with an invitation to participate in the research.

We will identify experts by attending symposia and/or conferences in relevant fields.

\section{Experts identified from practice (route B)}

We will identify practice-based experts through peers and through letters to relevant professional associations (listed below). These associations will be approached to disseminate letters of invitation to their members. Practice-based experts are defined as people who have worked for at least 5 years in a field relevant to geriatric pharmacotherapeutics.

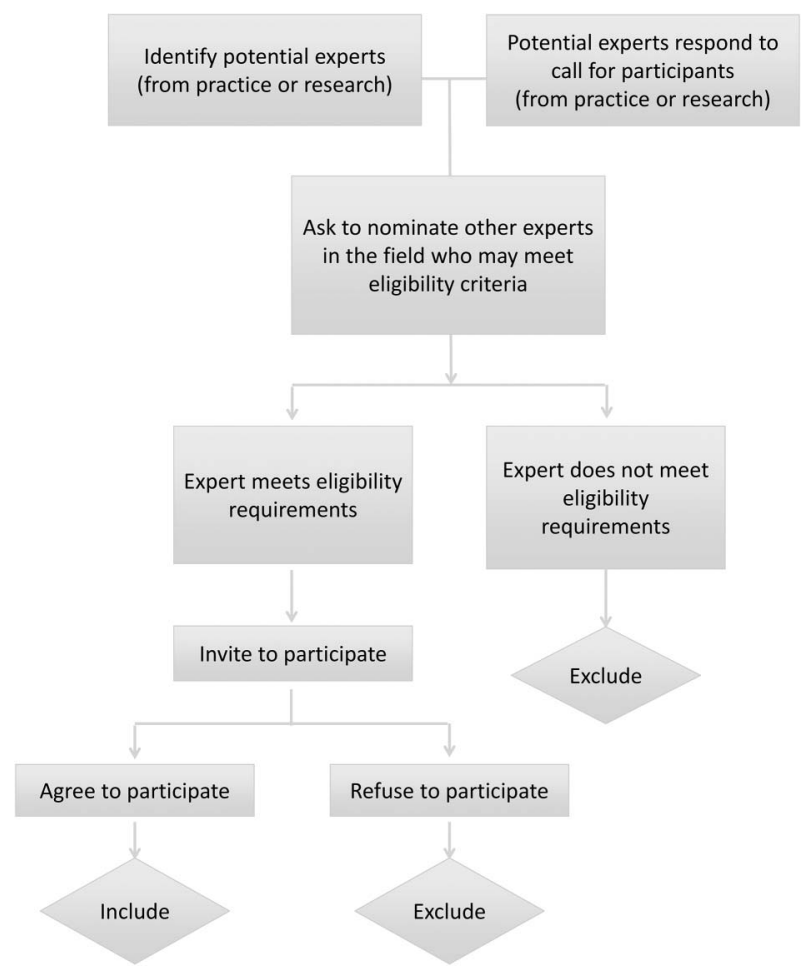

Figure 1 Recruitment process.
Relevant professional associations and networks will be approached to request their support to distribute an advertisement for recruitment to the expert panel.

\section{Screen for eligibility}

All identified experts will be invited to participate. The potential participants will receive materials to inform them of the study objectives and design, and the commitment required for participation. They will be sent an invitation letter, participant information sheet and a participant consent form. Experts will be screened at this point for eligibility to participate and also asked to nominate peers that may be interested in participating.

Potential participants will be asked to declare any conflicts of interest that may exist. Participants will be asked to commit to participate in all rounds of the Delphi technique required to develop the prescribing criteria. Potential participants will be provided with a clear explanation of the anticipated process, and an explanation that participation would be required over a period of months with two or more rounds of questioning with feedback. They will be asked to sign a written consent form to be involved in the Delphi technique process.

\section{Panel size and composition}

Delphi technique panels vary in size from 10 to over 1000, with no agreement or criteria in the literature around the ideal size. ${ }^{42}$ International explicit prescribing criteria developed using a Delphi technique have recorded expert panels between 6 and 99 members. ${ }^{38} 4344$ A minimum number of 20 participants will be set. The upper level will be fluid as larger panels increase the reliability of the outcomes. ${ }^{42}{ }^{45}$ It is more important to include relevant and interested experts than to set a definitive maximum number. ${ }^{45}$ Consequently, we will invite experts to participate who meet the inclusion criteria and have a minimum of 20 participants with no upper limit.

\section{Anonymity}

The iterative nature of a Delphi technique means that participants are anonymous to each other but not to the researcher. ${ }^{46}{ }^{47}$ This has been termed quasi-anonymity. ${ }^{47}$ At the conclusion of the process, participants who have responded in each round will be offered the choice to remain anonymous, or receive acknowledgement in the publication for their participation. ${ }^{48}$

\section{Design and content of the survey}

The primary questionnaire will consist of open-ended questions designed to elicit opinions about pharmacotherapeutics and quality use of medicines for people living with dementia. The questionnaire will be developed in Qualtrics: Online Survey Software and Insight Platform. ${ }^{49}$ Each round of the survey will be accompanied by a cover sheet that states the intentions of the round and for the second and subsequent rounds it will provide detailed and individualised feedback about responses to previous rounds. 


\section{Survey development}

The survey questions will be developed by an investigator (AP, a clinical pharmacist) and piloted with the other investigators (two academic pharmacists, a general practitioner, and a geriatrician). Adjustment will be made to the questions and format of the survey based on their feedback. The pilot process will be repeated with a small group of pharmacists' based on their feedback, the survey will be further adjusted.

\section{Definition of consensus}

The questions for rounds two and beyond will be scored on a five-point Likert scale. A median score and IQRs will be calculated for each item. Consensus on an individual statement is defined as an IQR equal to or less than 1.

\section{Enhancing response rate}

Attrition and participant fatigue is a common problem in Delphi techniques. A minimum of $70 \%$ response rate in each round to reduce response bias. ${ }^{42}$ We will aim to achieve $100 \%$ response rate, though will accept a round as valid if there is a $70 \%$ response rate.

Several methods will be used to improve attrition and response rate. Initially, face to face or telephone interviews will be conducted initially to develop rapport and respond to initial questions. ${ }^{46}{ }^{47}$ In each round, participants will be asked to return the questionnaire within 10 days after each round. After 7 days, participants will be sent a postcard as a reminder or thank you. After 14 days, the survey will be resent.

\section{Rounds}

A minimum of two rounds will be undertaken. The minimum of two rounds allows participants to have feedback and revise previous responses (see figure 2). Participant fatigue and attrition frequently limits the technique to a maximum of four rounds. ${ }^{47}$ It is anticipated that between two and four rounds will be undertaken, and a decision will be made during the research as to the appropriate point to stop, due to the need to balance a high response rate and the need to achieve consensus.

For the overall study after round 2, we have defined the point at which the study is ceased and there are no further rounds (see figure 2). Additionally, we have set the criteria for dropping a question in the second and subsequent rounds (see figure 2).

\section{First round}

The round 1 questionnaire will be a qualitative round asking open-ended questions with respect to pharmacotherapeutics for people living with dementia (see online supplementary file 1 ). The participants will be asked their opinion on medicines that are both preferred and potentially inappropriate for people living with dementia. There will be an option to provide qualifiers or elaborate on any statements.

A content analysis of the qualitative data collected during the first round will be analysed after the response from each participant is inputted into nVivo Qualitative Data Analysis software V.10 for Windows. ${ }^{50}$ Two researchers will work independently to code the data using content analysis to organise the data into themes. They will then collaborate to discuss any disagreement to reach a consensus. Statements that are the same or very similar in meaning will be amalgamated, and where relevant comments will be grouped and ordered therapeutically using the Anatomical Therapeutic Chemical (ATC) codes. ${ }^{46}$

\section{Second and subsequent results rounds}

We will ask the participant to state the extent to which they agree with the statements using a five-point Likert scale. They will also be asked several open-ended questions relating to the medicines.

For medicines or medicine classes that the participant has rated as inappropriate, the participant will be asked: (A) preferred therapeutic alternatives, (B) the reason it is inappropriate, (B) if possible, methods to minimise the risk and (D) if it is inappropriate only in an interaction with another drug or diagnosis.

For medicines identified as a preferred medicine, the participants will be asked (A) appropriate dose ranges and frequency, (B) indications or scenarios under which its absence is under-prescribing (ie, its absence is considered inappropriate).

The quantitative data (responses to the Likert scales) will be entered into SPSS V.22 for Macintosh statistical software for analysis. ${ }^{51}$ Descriptive statistics will be undertaken on the entire dataset after entering the data to provide a percentage of the overall response to each question. The median and IQR will be reported for each item.

Any responses to open-ended questions will be qualitatively analysed for content. The responses will be inputted into nVivo Qualitative Data Analysis Software V.10 for Windows and thematically coded. ${ }^{50}$ Statements that are the same or very similar will be amalgamated, and all comments will be grouped thematically.

The analysis will be provided as feedback to the experts. Consensus will be considered achieved on an individual statement if the IQR is $\leq 1$.

\section{Feedback reports}

Round 1 responses will be amalgamated with a literature review where possible to assist with a structured debate, as recommended by Hasson et al. ${ }^{46}$

If the study progresses to a third (and subsequent) round, we will send each panel member a cover letter and feedback from the preceding round. The feedback will consist of a summary of the group and the individual's responses will be sent to each participant after 


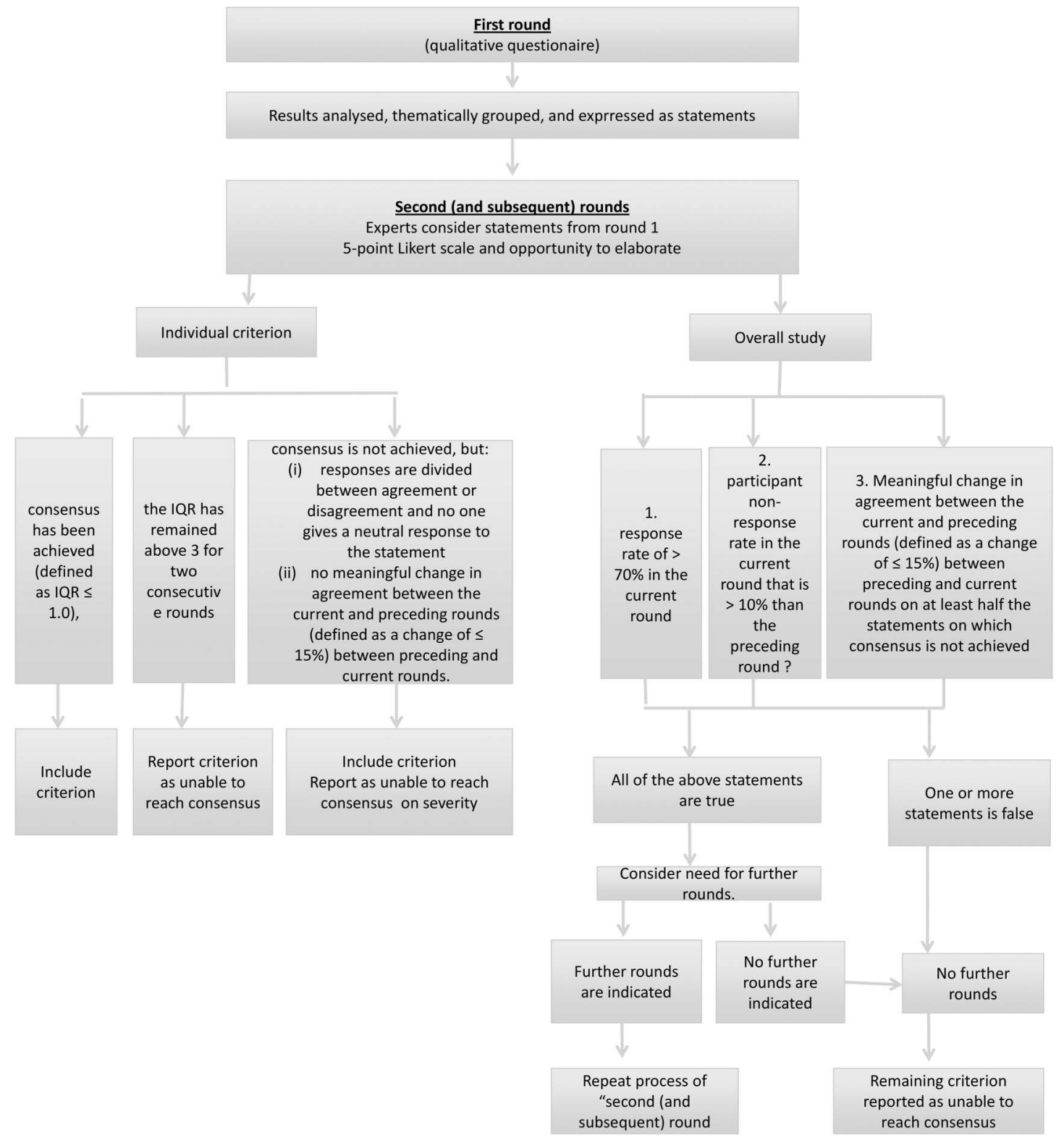

Figure 2 Study flow for the progression through the rounds.

every round. The summary will include (1) the median and mode for each item, (2) the participant's response in the previous round, (3) an indication of the current level of consensus (based on the IQR), and (4) a summary of participants responses as to why they have ranked an item in the way they did.

\section{Literature review (evidence base)}

Although there is insufficient research available to develop evidence-based guidelines, there is some available evidence. The proposed explicit consensus-based prescribing criteria in the final paper will reference the available evidence where possible. This will be achieved by the research steering group undertaking a detailed literature review for the use of the particular drug class in dementia following the recommendations and processes set out by the GRADE handbook. ${ }^{52}$ An evidence table will be created in GradePro Guideline Development Tool software to summarise the available evidence using their standard format. ${ }^{53}$ It will be used to provide a level and grade of evidence for each criterion, or to state that no evidence is available.

The search strategy will use the terms: (dementia OR ((cognitiv*) NEAR/2 (impair* OR dysfunction* OR disfunction* OR declin* OR disorder))) combined with identified drug classes or medicines. Articles will be limited to those published in English since 2000.

\section{Ethical considerations}

The study will be conducted in accordance with principles of the 'Declaration of Helsinki', Good Clinical Practice, and within the laws and regulations of Australia. 


\section{DISCUSSION}

This paper details the design of a study using the Delphi technique to address the issue of appropriate prescribing for older people living with dementia. The study aims to elicit opinion and gain consensus on appropriate medicine use for people living with dementia in Australia. The results of the study will be to create a consensus-based list of explicit prescribing criteria for people living with dementia in Australia. The outcomes of this study have the potential to improve prescribing for older people living with dementia, and to help clinicians identify appropriate prescribing.

There are both methodological strengths and weaknesses to using the Delphi technique for this study. Feedback from expert panel members by writing comments and rating items will elicit their opinion and work towards gaining consensus on appropriate medicine use for people living with dementia. Strengths of the Delphi technique include the participation of experts from geographically diverse regions of Australia. Participants remain anonymous to each other, and no individual could dominate discussion.

The Delphi technique is a technique to make use of the available information by summarising existing knowledge and experience of prescribing from an interprofessional panel of experts in geriatric practice. Consensus development methods, including the Delphi technique, are not a method to create new evidence. The results of this study can be used in clinical practice where it may be useful to support prescribing for older people living with dementia.

Contributors All authors shared the study conception. AP led the detailed protocol planning and drafted the manuscript. All authors critically reviewed the study protocol, and assisted in the development and implementation of the study. All authors read, revised and accepted the final draft.

Funding This research received no specific grant from any funding agency in the public, commercial or not-for-profit sectors. This work forms part of AP's $\mathrm{PhD}$, which is supported by a University Postgraduate Award by the University of Western Australia. KP is supported by an NHMRC Early Career Fellowship.

Competing interests None declared.

Ethics approval Ethical approval has been granted from the University of Western Australia's Human Research Ethics Committee (HREC) (reference: $\mathrm{RA} / 4 / 1 / 7172)$

Provenance and peer review Not commissioned; externally peer reviewed.

Data sharing statement Data used to develop the tables and figures presented in this article are available by emailing the corresponding author, AP, amy.page@uwa.edu.au.

Open Access This is an Open Access article distributed in accordance with the Creative Commons Attribution Non Commercial (CC BY-NC 4.0) license, which permits others to distribute, remix, adapt, build upon this work noncommercially, and license their derivative works on different terms, provided the original work is properly cited and the use is non-commercial. See: http:// creativecommons.org/licenses/by-nc/4.0/

\section{REFERENCES}

1. Larson EB, Shadlen MF, Wang L, et al. Survival after initial diagnosis of Alzheimer disease. Ann Intern Med 2004;140:501-9.

2. Wolfson $\mathrm{C}$, Wolfson $\mathrm{DB}$, Asgharian $\mathrm{M}$, et al. A reevaluation of the duration of survival after the onset of dementia. $N$ Engl J Med 2001;344:1111-16.
3. Australian Institute of Health and Welfare (AlHW). Dementia in Australia Canberra, Australia: AlHW, 2012.

4. Brookmeyer R, Johnson E, Ziegler-Graham K, et al. Forecasting the global burden of Alzheimer's disease. Alzheimers Dement 2007;3:186-91.

5. Schubert CC, Boustani M, Callahan CM, et al. Comorbidity profile of dementia patients in primary care: are they sicker?. J Am Geriatr Soc 2006;54:104-9.

6. Kunik ME, Snow AL, Molinari VA, et al. Health care utilization in dementia patients with psychiatric comorbidity. Gerontologist 2003;43:86-91.

7. Duthie A, Chew D, Soiza RL. Non-psychiatric comorbidity associated with Alzheimer's disease. QJM 2011;104:913-20.

8. Drewes YM, den Elzen WP, Mooijaart SP, et al. The effect of cognitive impairment on the predictive value of multimorbidity for the increase in disability in the oldest old: the Leiden 85-plus study. Age Ageing 2011;40:352-7.

9. Formiga F, Fort I, Robles MJ, et al. Comorbidity and clinical features in elderly patients with dementia: differences according to dementia severity. J Nutr Health Aging 2009;13:423-7.

10. Hoffmann $\mathrm{F}$, van den Bussche $\mathrm{H}$, Wiese $\mathrm{B}$, et al. Impact of geriatric comorbidity and polypharmacy on cholinesterase inhibitors prescribing in dementia. BMC Psychiatry 2011;11:190.

11. Somers M, Rose E, Simmonds D, et al. Quality use of medicines in residential aged care. Aust Fam Physician 2010;39:413-16.

12. Giron MS, Wang HX, Bernsten $C$, et al. The appropriateness of drug use in an older nondemented and demented population. $J \mathrm{Am}$ Geriatr Soc 2001;49:277-83.

13. Welsh TJ, Gladman JR, Gordon AL. The treatment of hypertension in people with dementia: a systematic review of observational studies. BMC Geriatr 2014:14:19.

14. McLean AJ, Le Couteur DG. Aging biology and geriatric clinical pharmacology. Pharmacol Rev 2004;56:163-84.

15. Hilmer SN, McLachlan AJ, Le Couteur DG. Clinical pharmacology in the geriatric patient. Fundam Clin Pharmacol 2007;21:217-30.

16. Hilmer SN. ADME-tox issues for the elderly. Expert Opin Drug Metab Toxicol 2008:4:1321-31.

17. Van Spall HGC, Toren A, Kiss A, et al. Eligibility criteria of randomized controlled trials published in high-impact general medical journals: a systematic sampling review. JAMA 2007;297:1233-40.

18. Farrall AJ, Wardlaw JM. Blood-brain barrier: ageing and microvascular disease-systematic review and meta-analysis. Neurobiol Aging 2009;30:337-52.

19. Lindblad $\mathrm{Cl}$, Hanlon JT, Gross $\mathrm{CR}$, et al. Clinically important drug-disease interactions and their prevalence in older adults. Clin Ther 2006;28:1133-43.

20. McCormick WC, Kukull WA, Van Belle G, et al. Symptom patterns and comorbidity in the early stages of Alzheimer's disease. J Am Geriatr Soc 1994;42:517-21.

21. Chang-Quan H, Hui W, Chao-Min W, et al. The association of antihypertensive medication use with risk of cognitive decline and dementia: a meta-analysis of longitudinal studies. Int J Clin Pract 2011:65:1295-305.

22. Stewart WF, Kawas C, Corrada M, et al. Risk of Alzheimer's disease and duration of NSAID use. Neurology 1997;48:626-32.

23. Ott A, Stolk RP, Van Harskamp F, et al. Diabetes mellitus and the risk of dementia: the Rotterdam Study. Neurology 1999;53:1937-42.

24. Jick H, Zornberg GL, Jick SS, et al. Statins and the risk of dementia. Lancet 2000;356:1627-31.

25. In t' Veld BA, Ruitenberg A, Hofman A, et al. Nonsteroidal antiinflammatory drugs and the risk of Alzheimer's disease. $N$ Engl $J$ Med 2001;345:1515-21.

26. Chen $\mathrm{Y}$, Zhou $\mathrm{K}$, Wang $\mathrm{R}$, et al. Antidiabetic drug metformin (Glucophage $(\mathrm{R})$ ) increases biogenesis of Alzheimer's amyloid peptides via up-regulating BACE1 transcription. Proc Natl Acad Sc U S A 2009;106:3907-12.

27. Holmes HM, Min LC, Yee M, et al. Rationalizing prescribing for older patients with multimorbidity: considering time to benefit. Drugs Aging 2013;30:655-66.

28. Holmes HM, Hayley DC, Alexander GC, et al. Reconsidering medication appropriateness for patients late in life. Arch Intern Med 2006;166:605-9.

29. van de Glind EM, van Enst WA, van Munster BC, et al. Pharmacological treatment of dementia: a scoping review of systematic reviews. Dement Geriatr Cogn Disord 2013;36:211-28.

30. Reisberg B, Borenstein J, Salob SP, et al. Behavioral symptoms in Alzheimer's disease: phenomenology and treatment. J Clin Psychiatry 1987;48(Suppl):9-15.

31. Lyketsos CG, Steinberg M, Tschanz JT, et al. Mental and behavioral disturbances in dementia: findings from the cache county study on memory in aging. Am J Psychiatry 2000;157:708-14. 
32. Bergh S, Selbæk G, Engedal K. Discontinuation of antidepressants in people with dementia and neuropsychiatric symptoms (DESEP study): double blind, randomised, parallel group, placebo controlled trial. BMJ 2012;344:e1566.

33. Seitz DP, Gill SS, Herrmann N, et al. Pharmacological treatments for neuropsychiatric symptoms of dementia in long-term care: a systematic review. Int Psychogeriatr 2013;25:185-203.

34. Schneider LS, Dagerman KS, Insel P. Risk of death with atypical antipsychotic drug treatment for dementia: meta-analysis of randomized placebo-controlled trials. JAMAn 2005;294:1934-43.

35. Laroche ML, Charmes JP, Merle L. Potentially inappropriate medications in the elderly: a French consensus panel list. Eur J Clin Pharmacol 2007;63:725-31.

36. Holt S, Schmiedl S, Thürmann PA. Potentially inappropriate medications in the elderly: the PRISCUS list. Dtsch Arztebl Int 2010;107:543-51.

37. Gallagher P, O'Mahony D. STOPP (Screening Tool of Older Persons' potentially inappropriate Prescriptions): application to acutely ill elderly patients and comparison with Beers' criteria. Age Ageing 2008;37:673-9.

38. Beers $\mathrm{MH}$. Explicit criteria for determining potentially inappropriate medication use by the elderly an update. Arch Intern Med 1997;157:1531-6.

39. Barry PJ, Gallagher P, Ryan C, et al. START (screening tool to alert doctors to the right treatment)-an evidence-based screening tool to detect prescribing omissions in elderly patients. Age Ageing 2007;36:632-8.

40. Hsu CC, Sandford BA. The Delphi technique: making sense of consensus. Pract Assess Res Eval 2007;12:1-8.

41. McKenna HP. The Delphi technique: a worthwhile research approach for nursing? J Adv Nurs 1994;19:1221-5.
42. Akins RB, Tolson $\mathrm{H}$, Cole BR. Stability of response characteristics of a Delphi panel: Application of bootstrap data expansion. BMC Med Res Methodol 2005;5:37.

43. Campbell SM, Cantrill JA, Roberts D. Prescribing Indicators For UK General Practice: Delphi consultation study. BMJ 2000;321:425-8.

44. Rognstad S, Brekke M, Fetveit A, et al. The Norwegian General Practice (NORGEP) criteria for assessing potentially inappropriate prescriptions to elderly patients. Scand J Prim Health Care 2009;27:153-9.

45. Powell C. The Delphi technique: Myths and realities. J Adv Nurs 2003;41:376-82.

46. Hasson F, Keeney S, McKenna H. Research guidelines for the Delphi survey technique. J Adv Nurs 2000;32:1008-15.

47. Keeney S, Hasson F, McKenna H. Consulting the oracle: ten lessons from using the Delphi technique in nursing research. $J \mathrm{Adv}$ Nurs 2006;53:205-12.

48. Rowe G, Wright G. The Delphi technique: Past, present, and future prospects-Introduction to the special issue. Technol Forecast Soc Change 2011;78:1487-90.

49. Qualtrics: Online Survey Software \& Insight Platform [program]. 61093 version. Provo, Utah, USA, 2015.

50. NVivo qualitative data analysis software [program]. 10 version, 2012

51. IBM SPSS Statistics for Windows, Version 22.0. [program]. Armonk, NY: IBM Corp., 2013.

52. Atkins D, Best D, Briss PA, et al., GRADE Working Group. Grading quality of evidence and strength of recommendations. $B M J$ 2013;328:1490-8

53. GRADEpro Guideline Development Tool (G2DT) [program]. 2.xx version, 2014. 